\title{
Suchtmittelassoziierte Gesundheitsstörungen - Tabakrauchen und Alkoholkonsum
}

\author{
U. John
}

Institut für Epidemiologie und Sozialmedizin der Universität Greifswald

\section{Prevention of substance-related diseases - tobacco smoking and alcohol consumption}

The prevalence of alcohol dependence in a German general hospital was estimated to be $17.2 \%$. Among the in-patients there were $34.7 \%$ daily smokers among females and $49.8 \%$ among males. Screening of all patients for tobacco smoking and alcohol risk drinking is essential to overcome these most preventable single health risks.

\section{Definition}

Gesundheitsstörungen aufgrund von Tabakrauchen und Alkoholkonsum sind alle Erkrankungen, für die aus epidemiologischen Befunden ein erhöhtes relatives Risiko im Zusammenhang mit dem Gebrauch dieser Substanzen (attributables Risiko) bekannt ist. Zu den $\mathrm{Ge}-$ sundheitsschädigungen, die aus dem Tabakrauchen entstehen, trägt das $\mathrm{Ni}$ kotin durch Gewöhnung und Abhängigkeit bei. Erkrankungen aufgrund von Alkohol sind bei mindestens riskantem Konsum zu erwarten. Es handelt sich um ein Trinken in Mengen, für die ein erhöhtes relatives Mortalitätsrisiko gilt: $20 \mathrm{~g}$ Reinalkohol pro Tag bei Frauen und $30 \mathrm{~g}$ bei Männern (Bühringer et al., 2000, Nomos Baden-Baden). Schädlicher Alkoholgebrauch und Alkoholabhängigkeit sind in den internationalen Krankheitsklassifikationssystemen ICD10 und DSM-IV definiert (Dilling et al., 1991, Huber Bern): Eine Alkoholabhängigkeit liegt vor, wenn mindestens drei der acht Kriterien erfüllt sind:

1. starker Wunsch oder eine Art Zwang, Alkohol zu konsumieren,

2. verminderte Kontrollfähigkeit, den Alkoholkonsum zu beenden,

3. Alkoholkonsum mit dem Ziel, Entzugssymptome zu lindern,

4. körperliches Entzugssyndrom,

5. Nachweis einer Toleranz,

6. eingeengtes Trinkverhalten, d. h. Alkoholkonsum eher nach dem psychischen oder körperlichen Bedarf als nach äußeren Anlässen,

7. Vernachlässigung von Interessen zugunsten des Alkoholkonsums,

8. anhaltender Konsum trotz Nachweises der negativen Folgen.
Schädlicher Gebrauch liegt vor, wenn der Konsum zu nachweisbaren Schäden führt.

\section{Aktuelle Situation}

Gesundheitsstörungen aufgrund von Tabakrauchen oder Alkoholkonsum bilden einen erheblichen Anteil im Ursachengeschehen von Mortalität und Morbidität (John \& Hanke, Eur J Cancer Prev, i. Dr.). Beim Tabakrauchen besteht für mehr als 300 Erkrankungen oder Todesursachen ein erhöhtes attributables Risiko (s. a. nächste Folge dieser Serie in DMW 6/2002). Für einen Teil sind ursächliche Beziehungen belegt. Bei riskantem Alkoholkonsum handelt es sich um mehr als 180 Erkrankungen (ICD vierstellig) und zusätzlich Unfälle oder Verletzungen. Ein Fünftel der Gesamtmortalität ist in Deutschland auf Tabakrauchen und Alkoholkonsum rückführbar (John \& Hanke, Eur J Cancer Prev, i. Dr.). Beide Verhaltensweisen bilden zusammen die bedeutendste verhaltensbezogene Todesursache ( $\mathrm{J}$ Amer Med Ass 1993; 270: 2207-2212; Abb. 1).

Daten aus der Erwachsenenbevölkerung einer Region in Norddeutschland zeigen, dass von den Rauchern lediglich 1,9\% Entwöhnungskurse und 8,1\% Präparate zur Abstinenzerlangung, insbesondere Nikotinsubstitutionspräparate, in Anspruch nahmen (Sucht 2000; 46: 398-407). Von den gegenwärtig Alkoholabhängigen hatten $70,9 \%$ keinerlei Kontakt zur Suchtkrankenhilfe, aber 80,0\% der Alkoholabhängigen gaben an, mindestens einmal im Jahr einen praktischen Arzt oder Arzt für Allgemeinmedizin zu konsultieren (Sucht 2000; 46: 9-17). Systematisch durchgeführte
Prävention in der medizinischen Versorgung lässt einen signifikanten Beitrag zur Senkung der Morbidität erwarten. Ein wesentliches Mittel dazu bilden Früherkennung und Frühbehandlung.

\section{Frühkennung und Frühbehandlung}

Die Prävention suchtmittelassoziierter Gesundheitsstörungen lässt einen erheblichen Beitrag zur Erhöhung der Lebenserwartung und Senkung der Morbidität erwarten. Um das zu erreichen, ist neben Verhaltensregeln im Gemeinwesen die individuelle Motivierung ein wesentlicher Weg zu Abstinenz oder Mäßigung. Dabei sind die Ärztinnen und Ärzte von zentraler Bedeutung. In

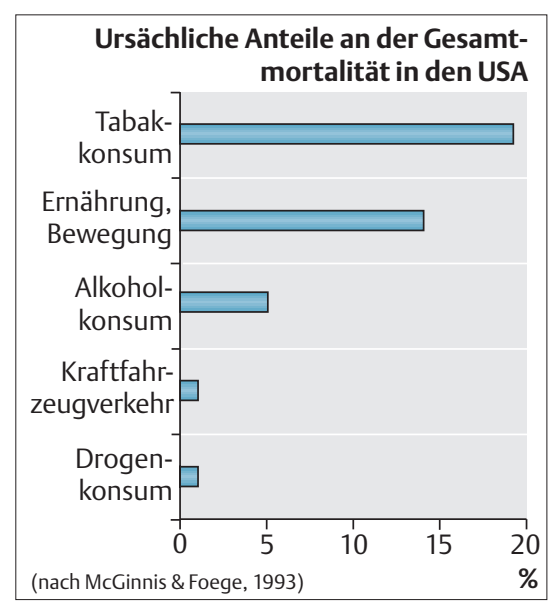

Abb. 1: Tabak- und alkoholattributable Mortalität

der medizinischen Versorgung sind besonders Interventionen sinnvoll, die frühzeitig in der Entwicklung von Problemen im Umgang mit psychotropen Substanzen einsetzen. Solche Frühinterventionen erfordern zwei Bestandteile: erstens ein Screening und zweitens die Intervention selber.

Für das Screening eignen sich Patientenaussagen, zum Rauchen der FagerströmTest für Nikotinabhängigkeit mit sechs Fragen (FTND; Schoberberger \& Kunze, 


\section{Studien zur Prävalenz der Alko- holabhängigkeit}

In einer repräsentativen Untersuchung der Prävalenz von schädlichem Alkoholkonsum und Alkoholabhängigkeit unter Krankenhauspatienten wurde bei allen Patienten ein Screening und im positiven Fall ein standardisiertes Interview durchgeführt, das zu ICD10-Diagnosen führte (John et al., 1996, Nomos BadenBaden). Die Untersuchung zeigte für ein städtisches Allgemeinkrankenhaus in Lübeck (mit internistischer und chirurgischer Abteilung) für die internistische Abteilung folgende Prävalenzen für Alkoholstörungen unter den 635 Patienten im Alter von 18 bis 64 Jahren (Alc Alcoh 1999; 34: 786-794; Tab. 1).

Tab. 1 Prävalenzen für Alkoholstörungen

\begin{tabular}{llll}
\hline Diagnose & Frauen & Männer & Gesamt \\
\hline $\begin{array}{l}\text { Abhängigkeit } \\
\begin{array}{l}\text { Schädlicher } \\
\text { Konsum }\end{array}\end{array}$ & $8,7 \%$ & $23,7 \%$ & $17,2 \%$ \\
$\begin{array}{l}\text { Verdachts- } \\
\text { diagnose* }\end{array}$ & $2,2 \%$ & $8,8 \%$ & $3,5 \%$ \\
\hline
\end{tabular}

* Keine Diagnose der Abhängigkeit oder des schädlichen Konsums, aber mindestens ein Hinweis auf Alkoholkonsum als mögliche Ursache für aktuelle Erkrankung

In dem Einzugsgebiet des Krankenhauses fanden sich aufgrund einer repräsentativen Bevölkerungsuntersuchung 1,3\% aktuell Alkoholabhängige und weitere $1,1 \%$ Patienten mit schädlichem Alkoholkonsum. Unter älteren stationären Patienten im Allgemeinkrankenhaus (ab 65 Jahren) sind die Diagnosen einer Alkoholabhängigkeit oder eines Alkoholmissbrauches deutlich seltener: 5,5\% Alkoholabhängigkeit oder schädlicher Konsum, 2\% remittierte Alkoholabhängigkeit. In der Ambulanz des untersuchten Krankenhauses wiesen 33,8\% der Patienten eine positive ScreeningDiagnose auf (John et al., 1996, Nomos Baden-Baden).

In einer Studie zur Prävalenz alkoholbezogener Störungen aufgrund einer Zufallsauswahl von zwölf Allgemeinarztpraxen (929 Screening-Untersuchungen mit Patienten im Alter von 14 bis 75 Jahren) ergaben sich bei $12,1 \%$ der männlichen Patienten eine gegenwärtige Alkoholabhängigkeit und bei weiteren 5,6\% ein schädlicher Alkoholkonsum. Von den Frauen waren es 4,2\% mit gegenwärtiger Alkoholabhängigkeit und 2,1\% mit schädlichem Alkoholkonsum (John et al., 1996, Nomos BadenBaden).
1999, Springer Wien), zu schädlichem Alkoholkonsum oder Alkoholabhängigkeit der Lübecker Alkoholabhängigkeits- und -missbrauchs-Screening-Test mit sieben Fragen (LAST; Rump et al. 2001, Hogrefe Göttingen). Bei positivem Resultat kann eine standardisierte Diagnostik der Nikotin- oder Alkoholabhängigkeit gemäß den Kriterien der ICD-10 durchgeführt werden. Laborparameter (GGT, GOT, GPT, Crea, Urea, CDT) sollten nach Möglichkeit berücksichtigt werden, für die Einschätzung riskanten Konsums auch Trinkmenge und -häufigkeit. Die Laborparameter sind sinnvoll, um dem Patienten Alkoholfolgestörungen zu erläutern. In jedem Fall sollten dabei die Trinkgrenzen zu riskantem Konsum berücksichtigt werden.

Für die Kurzintervention eignen sich Mittel, die in weniger als 10 Minuten anwendbar sind, wie die ärztliche Kurzberatung gemäß der Motivierenden $\mathrm{Ge}$ sprächsführung, der ärztliche Ratschlag und Selbsthilfemanuale (Rumpf et al., 2000, in: Deutsche Hauptstelle gegen die Suchtgefahren, Hrsg., Freiburg, 211219). Entscheidend ist, dass auch Patienten mit geringer oder fehlender Änderungsmotivation erreicht werden.

Besonders geeignet ist die Motivierende Gesprächsführung. Sie lässt sich leicht erlernen. Ihr Ziel besteht in der Förderung der Motivation zu Abstinenz oder Mäßigung. Das erreicht der Arzt durch Fragen und Rückmeldungen an den $\mathrm{Pa}-$ tienten, die ihn zu einer Auseinandersetzung mit der Problematik motivieren (Hapke, 2000; Lambertus Freiburg). Es sollen ein vertrauensvolles Gespräch ermöglicht, der Kontakt zum Patienten stabilisiert, die Inanspruchnahme notwendiger Hilfen gefördert und emotionale sowie kognitive Voraussetzungen für eine Entscheidung zur Abstinenz geschaffen werden. Der Arzt sollte Versuche zur Reduktion der Rauch- oder Trinkmenge akzeptieren, da die entsprechenden Erfahrungen die Entwicklung des Patienten fördern. Entscheidend ist, dass sich der Patient nicht zurückzieht und den Arzt auch bei Erlebnissen des Scheiterns wieder aufsucht. Das Konzept ist besonders nützlich für Patienten, die keine Änderungsbereitschaft oder aber Ambivalenz zeigen.

Mittlerweile werden Fortbildungen in der Motivierenden Gesprächsführung durchgeführt. Sie ist Bestandteil der Ausbildung für die ärztliche Fachkunde

\section{Weitere Informationen}

Informationen für Ärzte zu Suchtmitteln sind erhältlich bei der Deutschen Hauptstelle gegen die Suchtgefahren (DHS) in Hamm (www.dhs.de).

Einrichtungen für Suchtprävention: Beratungsstellen für Menschen mit Suchtmittelproblemen (Adressen bei der DHS)

Sucht. Zur Zeit sind die betroffenen medizinische Fachverbände mit der Entwicklung von Leitlinien zur Behandlung von Suchterkrankungen beschäftigt, die Früherkennung und Frühintervention umfassen. Zur strukturellen Verankerung erwies sich im Allgemeinkrankenhaus ein Liaisondienst mit ständiger Präsenz eines Suchtberaters auf Station unter Aspekten der Prävention als überlegen gegenüber dem Konsiliarsystem (Addiction 1998; 93: 1777-1786).

Fazit: Es müssen sekundärpräventive Programme in Arztpraxen und Allgemeinkrankenhäusern geschaffen werden. Nach dem Stand des Wissens helfen sie, Kosten und stationäre sowie ambulante Behandlungen zu sparen. Aber auch heute kann der Arzt bereits durch vertretbaren Aufwand erheblich zur Senkung suchtmittelassoziierter Erkrankungen beitragen. 4

Prof. Dr. Ulrich John

Ernst-Moritz-Arndt-Universität Greifswald Institut für Epidemiologie und Sozialmedizin Walther-Rathenau-Straße 48 17487 Greifswald Internet: http://www.medizin.unigreifswald.de/epidem

In der nächsten Folge lesen Sie:

Anti-Tabak-Kampagnen

\section{Fachliche Betreuung der} "Serie ... Prävention॥:

Prof. Dr. Dr. h. c. Peter C. Scriba

Medizinische Klinik / Klinikum Innenstadt der Universität München

Ziemssenstr. 1

80336 München

Prof. Dr. Friedrich W. Schwartz

Medizinische Hochschule Hannover

Abteilung für Epidemiologie, Sozialmedizin und Gesundheitsforschung

Carl-Neuberg-Str. 1

30623 Hannover 